\title{
Proton and electron acceleration by quasi-perpendicular fast magnetosonic shocks in interplanetary space
}

\author{
G. M. Simnett ${ }^{1}$, J.-I. Sakai ${ }^{2}$, and R. J. Forsyth ${ }^{3}$ \\ 1 School of Physics \& Astronomy, University of Birmingham, Birmingham B15 2TT, UK \\ e-mail: gms@star.sr.bham.ac.uk \\ ${ }^{2}$ Laboratory for Plasma Astrophysics, Toyama University, Toyama 930-8555, Japan \\ 3 Blackett Laboratory, Imperial College of Science, Technology and Medicine, London SW7 2BZ, UK
}

Received 9 February 2004 / Accepted 30 May 2005

\begin{abstract}
Recent observations from the Ulysses spacecraft (Simnett 2003, Sol. Phys., 213, 387) have measured the energy spectrum of the ambient ions associated with the passage of a fast magnetosonic quasi-perpendicular shock driven by a coronal mass ejection (CME). Near the shock front the energy spectrum of the accelerated ions, which are predominantly protons, exhibits features (maxima) at about $250 \mathrm{keV}-1.5 \mathrm{MeV}$. We show that these are the right order of magnitude for protons which have been accelerated by the surfatron mechanism at a fast magnetosonic shock wave propagating perpendicular to the ambient magnetic field. The in situ measurements therefore illustrate directly that this mechanism could possibly be responsible for interplanetary particle acceleration. The shock is also accompanied by an increase in the near-relativistic electron intensity of almost two orders of magnitude. We present a similar event observed by the ACE spacecraft.
\end{abstract}

Key words. miscellaneous

\section{Introduction}

Charged particle acceleration is one of the most important processes occurring in the corona and throughout the heliosphere. Although both electron and ion acceleration are of interest, from an energetics viewpoint protons dominate and the most relevant energy region is typically from $\sim 0.1-10 \mathrm{MeV}$. A probable acceleration mechanism is via a wave-particle interaction in a travelling interplanetary magnetic disturbance, which we discuss in Sect. 2. We concentrate first on the ions, and secondly address the electrons.

Evidence of shock acceleration in the interplanetary medium has been available for many years (Ogilvie \& Arens 1971). However, since the early measurements knowledge of the particle distribution function has gradually been improving. Simnett (2003) has recently studied the energetic charged particles accelerated by a fast shock driven by a coronal mass ejection (CME) which passed over the Ulysses spacecraft on 10 May, 2001. The quality of the measurements was such that the particle anisotropy and energy spectrum was measured with a time resolution of either 12 or $24 \mathrm{~s}$ in an energy region from $\sim 50 \mathrm{keV}-4.5 \mathrm{MeV}$ (divided into eight logarithmicallyspaced channels). We give an example of a similar event observed by the Advanced Composition Explorer (ACE) spacecraft on 28 April, 2001.

Both Ulysses and ACE have on board plasma and magnetic field monitors, which enable us to estimate the parameters of the shock which are relevant to the acceleration theory. From these data we are able to calculate the likely energy spectrum of the accelerated ions and we show that some aspects of the theory match well with the observations for these events. However, it is plausible that there is more than one appropriate scale involved, and that theoretical predictions over a small scale may get modified, in a way yet to be addressed, over a larger scale. Thus a spacecraft observing an approaching or departing shock is observing over a much larger scale than when it is actually making in situ measurements as the shock passes.

The two events we discuss also had high intensities of electrons. Theoretically it has proved difficult to accelerate electrons at low Mach-number shocks, although Hoshino \& Shimada (2002) have had some success for high Mach-number shocks. This casts some doubt on the hypothesis that the shock is accelerating the electrons out of the solar wind plasma; an alternative interpretation would require some trapping to occur in the magnetic cloud behind the shock, possibly with some additional acceleration by the shock.

\section{The acceleration mechanism}

When an electrostatic wave travels through a region with a magnetic field, the ambient charged particles may experience some acceleration. In particular, the $\boldsymbol{V}_{\mathrm{p}} \times \boldsymbol{B}$ acceleration (here $\boldsymbol{V}_{\mathrm{p}}$ is the wave phase velocity and $\boldsymbol{B}$ the magnetic field) has received a great deal of attention (Sagdeev \& Shapiro 1973; 
Sugihara \& Midzuno 1979). These authors showed that a largeamplitude electrostatic wave propagating across an external magnetic field can trap some particles and accelerate them in the direction parallel to the wave front and perpendicular to the magnetic field. When they are accelerated to the $\boldsymbol{E} \times \boldsymbol{B}$ drift speed, they are detrapped, and this therefore provides an effective limit to the acceleration. The trapped particles reach the maximum speeds in a very short period although for a travelling interplanetary shock, this is probably not important. Hoshino (2001) and Hoshino \& Shimada (2002) have developed the shock surfing (surfatron) concept in more detail, especially for electrons at high Mach number shocks, by invoking magnetosonic solitons to trap the electrons so that they may be accelerated. Thus in reality the theoretical work may be too simplistic, in the sense that the actual shocks contain features that are too complex to address with the basic theory. However, it is useful to compare the theroretical work with the observations to see what common ground there is, recognising that an attempt to try to match theory and observation at the present time is bound to meet with limited success.

Ohsawa (1985) showed that quasi-perpendicular fast magnetosonic shocks accelerate trapped ions up to the speed $v \sim$ $V_{\mathrm{A}}\left(m_{\mathrm{i}} / m_{\mathrm{e}}\right)^{1 / 2}\left(M_{\mathrm{A}}-1\right)^{3 / 2}$, where $V_{\mathrm{A}}$ is the Alfvén speed, $m_{\mathrm{i}}$ the ion mass, $m_{\mathrm{e}}$ the electron mass, and $M_{\mathrm{A}}$ the Alfvén Mach number. Ohsawa \& Sakai $(1987,1988)$ applied this mechanism to the prompt particle acceleration in impulsive solar flares. The characteristics of the $\boldsymbol{V}_{\mathrm{p}} \times \boldsymbol{B}$ acceleration are summarized as follows:

1. It operates near the fast magnetosonic shock front propagating almost perpendicular to the magnetic field with low Alfvén Mach number like $M_{\mathrm{A}}<3$ in low $\beta$ plasma. Note however, that more complex theory (e.g. Hoshino 2001) can also make the surfatron mechanism work in shocks of high Mach number.

2. When $\omega_{\text {pe }}>\omega_{\text {ce }}$, only protons can be accelerated up to the speed $v \sim V_{\mathrm{A}}\left(m_{\mathrm{i}} / m_{\mathrm{e}}\right)^{1 / 2}\left(M_{\mathrm{A}}-1\right)^{3 / 2}$. Here $\omega_{\mathrm{pe}}$ is the electron plasma frequency and $\omega_{\mathrm{ce}}$ is the electron cyclotron frequency. When $\omega_{\mathrm{pe}}<\omega_{\mathrm{ce}}$, both electrons and ions can be accelerated (Ohsawa \& Sakai 1988).

3. The acceleration time is rapid and is given by $\tau_{\mathrm{A}}=$ $\omega_{\mathrm{ci}}{ }^{-1}\left(m_{\mathrm{i}} / m_{\mathrm{e}}\right)^{1 / 2}$. Here $\omega_{\mathrm{ci}}$ is the ion cyclotron frequency.

4. If the background plasma is relatively low $\beta$, the accelerated protons show a bump-on-tail distribution around the previous maximum velocity (Ohsawa \& Sakai 1985).

\section{The observations}

The observations we discuss are related to two strong shocks which were observed at Ulysses and ACE respectively. Both had very high proton (ion) and electron intensities; we first discuss the proton measurements, and then the electrons.

\subsection{Protons}

The starting point for this study is the arrival at Ulysses of a fast quasi-perpendicular shock early on 10 May, 2001. Figure 1

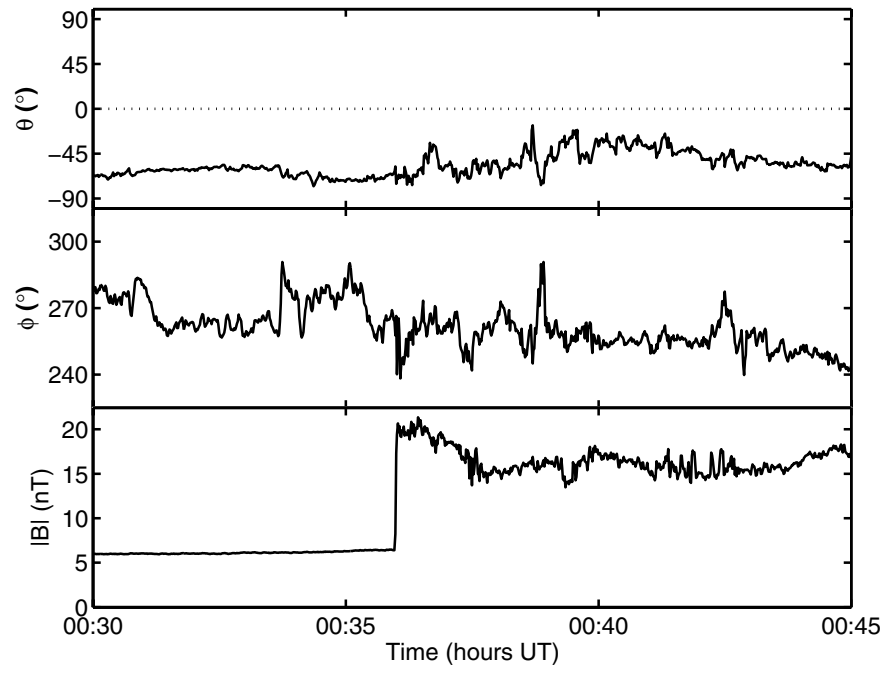

Fig. 1. The direction and magnitude of the interplanetary magnetic field at Ulysses early on 10 May, 2001. The data are plotted at a time resolution of $1 \mathrm{~s}$.

shows the three components of the magnetic field in RTN coordinates, plotted with 1-s time resolution, and the time of the shock arrival is 00:35:59 $\pm 1 \mathrm{~s}$ UT. There are two noteworthy points from this figure: (1) The magnetic field intensity increases in $\sim 2 \mathrm{~s}$ from the ambient background level of $6.4 \mathrm{nT}$ to $\sim 19.4 \mathrm{nT}$. (2) The azimuthal component $(\phi)$ of the field prior to the shock arrival is typically within $\sim 10^{\circ}$ of $270^{\circ}$, which is the direction perpendicular to the Earth-Sun line. Following the shock passage the angle changes by around $25^{\circ}$ for about $20 \mathrm{~s}$, and then gradually rotates to around $240^{\circ}$ by 00:45 UT. This clearly means that the shock may be classed as quasi-perpendicular. Deviations in the azimuth direction from $270^{\circ}$ could indicate the degree of turbulance in the plasma both sides of the shock. We have performed a coplanarity analysis using the data from $5 \mathrm{~s}$ either side of the shock transition, and the angle between the shock normal and the upstream magnetic field, $\theta_{\mathrm{Bn}}$ is $81.3^{\circ}$. However, this will be influenced by any foot and overshoot features created by the shock itself. We therefore did another analysis moving of the order of $10 \mathrm{~min}$ away from the shock either side and then using roughly $3 \mathrm{~min}$ of data to determine the average of the upstream/downstream fields. This gave an angle $\theta_{\mathrm{Bn}}$ of $54.1^{\circ}$. Thus the shock is only quasi-perpendicular. Conceptually, this might be expected if the shock was driven by a magnetic cloud centred near the ecliptic plane, which would be a likely evolution of the CMEs observed at the Sun.

We now turn to the plasma diagnostics. Figure 2 shows the proton density (top panel) and speed (middle panel) and temperature (bottom panel) for the first $6 \mathrm{~h}$ of 10 May. It is clear from the speed that Ulysses is experiencing a fairly constant high speed stream which is immediately behind the shock front. The density shows a modest increase at the shock. The temperature shows the largest increase, going from around $2 \times 10^{4} \mathrm{~K}$ to around $10^{6} \mathrm{~K}$. Unfortunately there was a data gap from 00:20-00:43 UT, which is the period covering the shock arrival. This means that the plasma parameters actually at the 

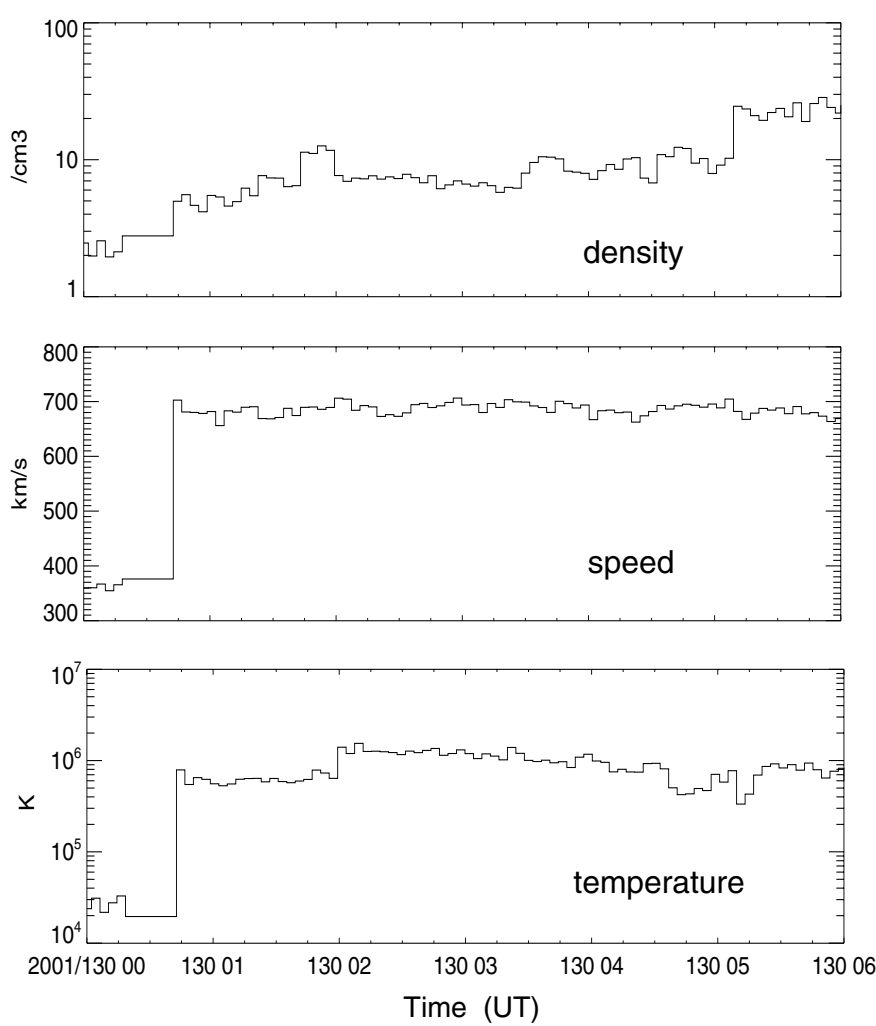

Fig. 2. The solar wind density, speed and temperature at Ulysses early on 10 May, 2001. These values are taken from the proton population and are $\sim 4$ min averages. Note the data gap from around 00:20-00:43 UT, which is the period covering the shock arrival. (Courtesy of the Ulysses Common Data File.)

shock itself are only estimates, based on extrapolation across the data gap.

The energetic particle measurements were made with the HI-SCALE instrument (Lanzerotti et al. 1992) on the Ulysses spacecraft. The event on 10 May, 2001 was noteworthy for the high intensities of electrons above $\sim 40 \mathrm{keV}$ and ions above $1 \mathrm{MeV}$ which appeared in association with the shock, which itself was driven by a well-observed coronal mass ejection (Simnett 2003). Although the electrons were intense, as seen by HI-SCALE, the spectrum was featureless, so we concentrate first on the ions, which are predominantly protons; we shall refer to them simply as protons. The Ulysses spacecraft spins every $12 \mathrm{~s}$, and the detectors accumulate data either over $45^{\circ}$ or $90^{\circ}$ of the spin. These intervals are referred to as sectors.

Figure 3 shows the proton energy spectrum for the period 00:36:35-00:36:47 UT, which covers just one spacecraft spin. The data cover the peak sector from each of two detectors sampling the protons, and it is clear that there is a pronounced peak in the energy interval from $207-336 \mathrm{keV}$. This is the energy interval for the $\mathrm{P}^{\prime} 4$ channel (see Lanzerotti et al. 1992, for a definition of the terminology and the orientation of the various sectors on the sky) but the energy interval for the P4 channel is similar. Figure 4 shows the intensity-time history of sector 5 of $\mathrm{P}^{\prime} 4$, and it shows a 40-fold intensity increase in the time interval immediately following the shock passage. The gaps in the record are a normal feature of the data, as the telemetry is

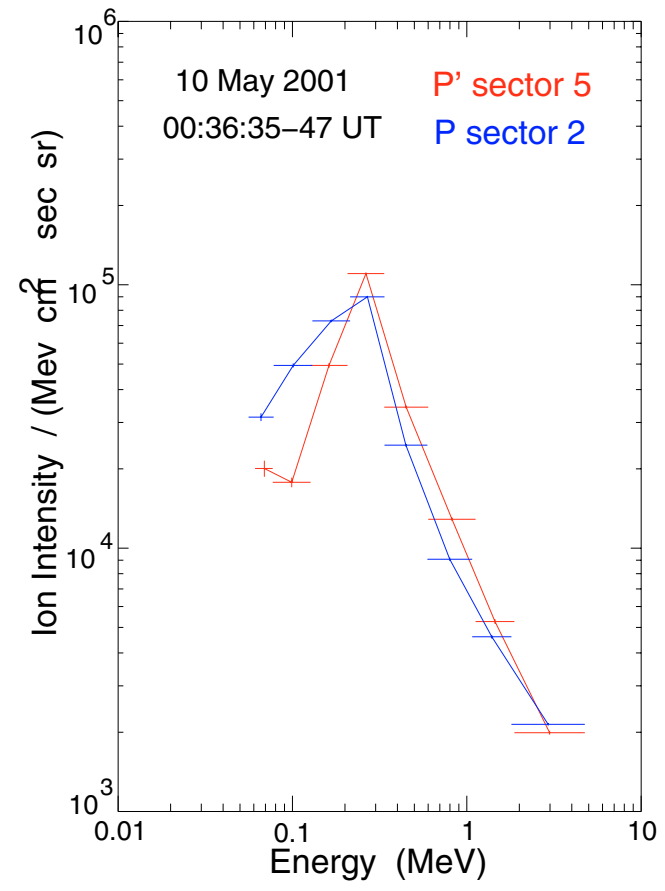

Fig. 3. The proton energy spectrum at the shock at Ulysses. (Simnett 2003.)

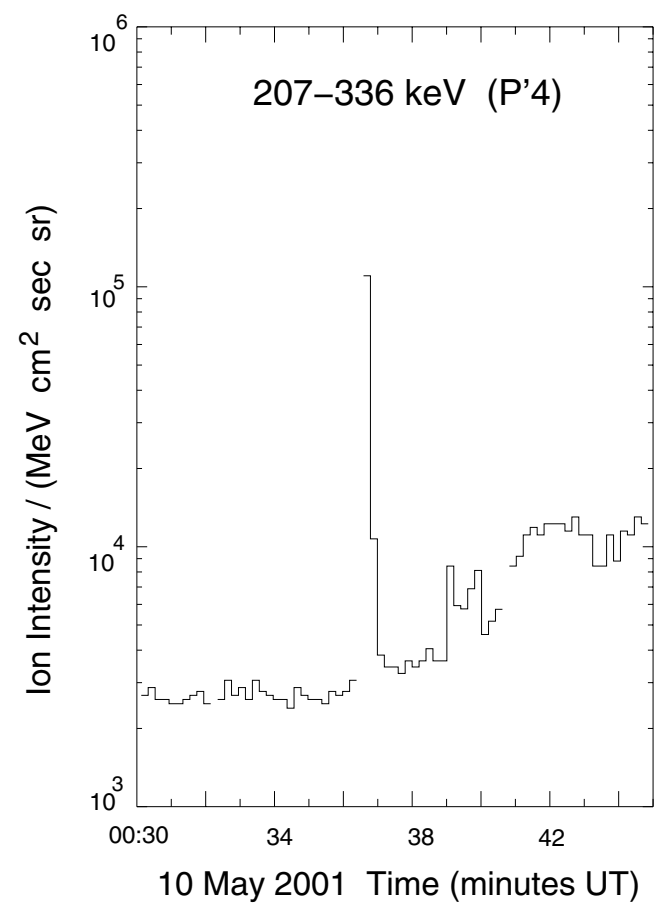

Fig. 4. The intensity-time history, at Ulysses and at 12-s resolution, of sector 5 of the 207-336 keV proton intensity from the LEMS120 telescope ( $\left.\mathrm{P}^{\prime} 4\right)$. (Simnett 2003.)

optimised to acquire as much data as possible, with the result that one spin out of 20 is missing.

Figure 3 shows the proton spectrum immediately after the passage of the shock. We have examined the data just upstream of the shock, and Fig. 5 shows that there are protons streaming away from the shock, with a pronounced spectral peak at just over $1 \mathrm{MeV}$. The left part of Fig. 5 shows the intensity-time 

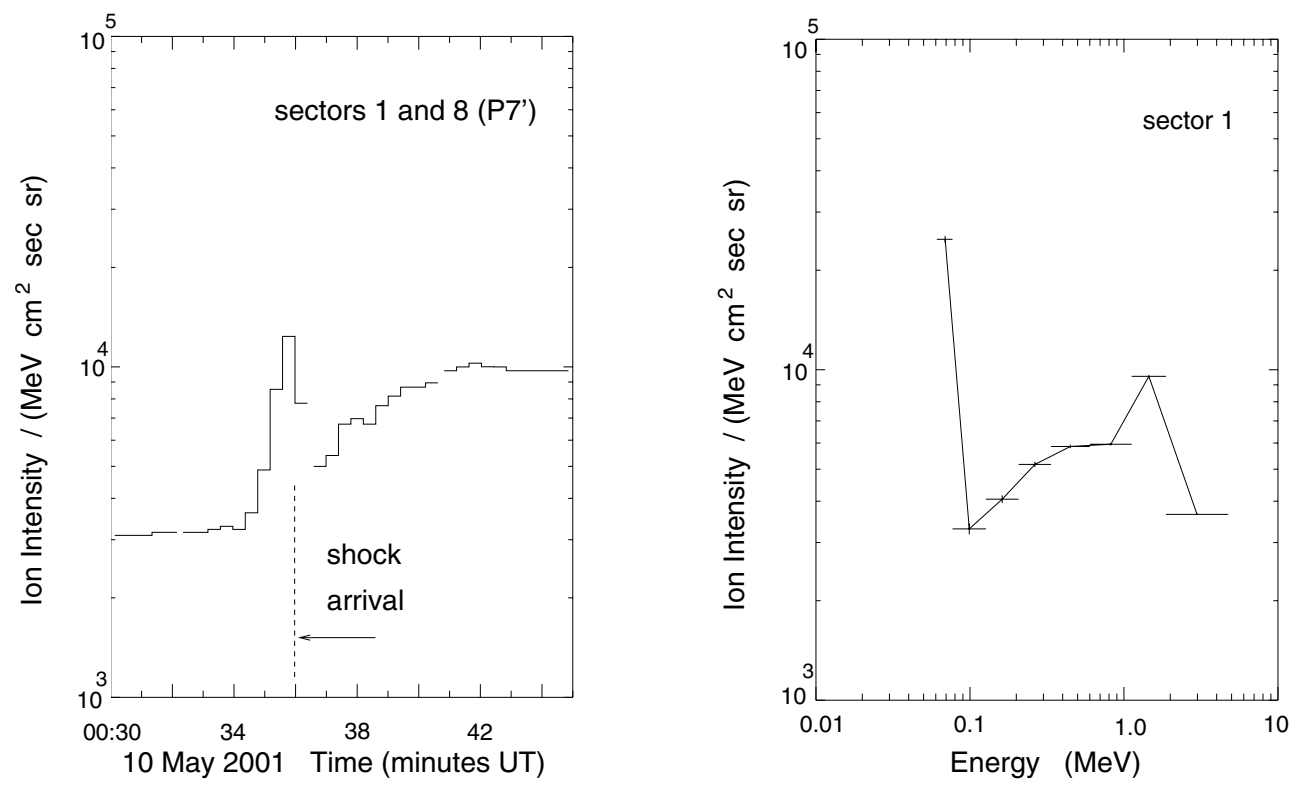

Fig. 5. The proton intensity-time history and energy spectrum just ahead of the shock at Ulysses. (Simnett 2003) The energy spectrum is from 00:34:49-00:36:11 UT.
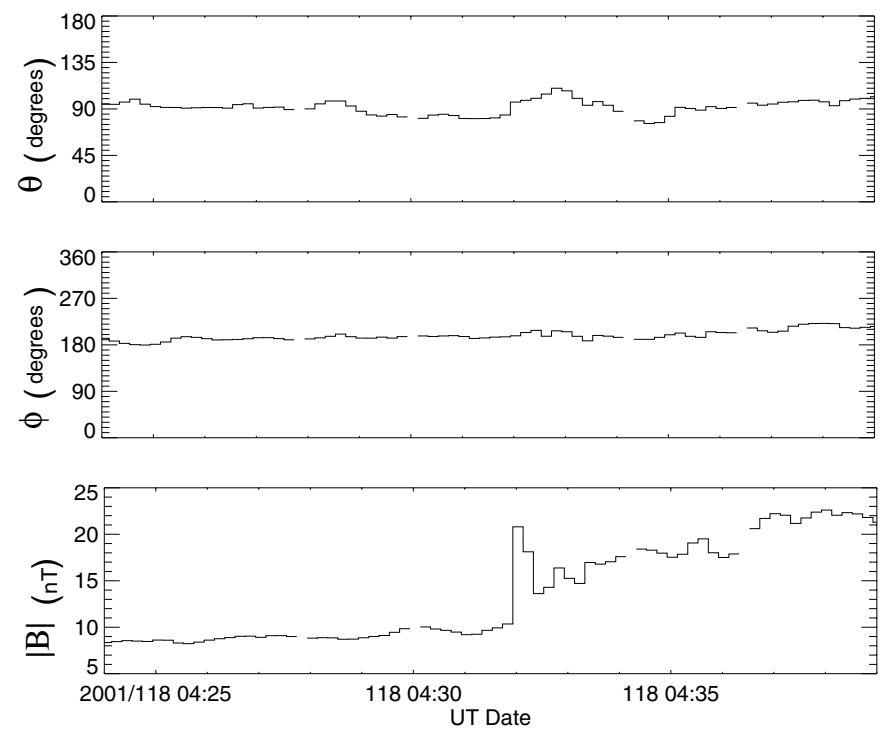

Fig. 6. The direction and magnitude of the interplanetary magnetic field at ACE early on 28 April 2001. The data are plotted at a time resolution of $12 \mathrm{~s}$. (Courtesy of Dr. C. W. Smith.)

history of the average of sectors 1 and 8 of the 1.123-1.874 $\mathrm{MeV}$ channel $\left(\mathrm{P}^{\prime} 7\right)$, while the right panel shows the energy spectrum accumulated over 6 spins from 00:34:49-00:36:11 UT. Simnett (2003) showed that the proton spectrum showed some evidence for these spectral features for two days prior to the shock arrival, and for several hours after its passage. There is considerable trapping occurring behind the shock front, as the proton intensity in the energy region $\sim 0.1 \mathrm{MeV}$ is enhanced by 2 orders of magnitude.

We have searched the Ulysses and ACE data for other examples of in situ measurements of the spectrum of accelerated protons at a travelling interplanetary shock. An example of such an event is that where a shock reached ACE at 04:32 UT on 28 April, 2001. The direction and magnitude of the magnetic field from 04:24-04:39 UT are shown in Fig. 6. This event was associated with a halo coronal mass ejection seen by LASCO (Brueckner et al. 1995) on the SOHO spacecraft, and a GOES class M7.8 X-ray flare, with a maximum at 13:12 UT, 26 April, from a location N17 W31 on the visible solar disc. The onset time, extrapolated back to a notional $1 R_{\odot}$, of the edge visible at a position angle of $37^{\circ}$ near the solar north pole is $\sim 11: 52 \mathrm{UT}$. The projected speed in the plane of the sky for this CME was around $960 \mathrm{~km} \mathrm{~s}^{-1}$ and there were type III radio bursts seen by the Waves instrument (Bougeret et al. 1995) on board the WIND spacecraft starting around 12:00 UT. Thus we are confident that the shock driven by this event would propagate to the Earth. On this premise the average propagation speed from the Sun to ACE is $1008 \mathrm{~km} \mathrm{~s}^{-1}$, which indicates that the actual speed near the Sun must have been significantly higher than the plane of the sky speed. As the solar wind speed measured at ACE following the shock passage was around $725 \mathrm{~km} \mathrm{~s}^{-1}$, then there must have been considerable deceleration in the interplanetary medium.

The following are courtesy of Dr. Qiang $\mathrm{Hu}$ of the ACE magnetometer team. The shock speed in the spacecraft frame is $905 \pm 59 \mathrm{~km} \mathrm{~s}^{-1}$ and in the plasma frame it is $492 \pm$ $36 \mathrm{~km} \mathrm{~s}^{-1}$. The density compression ratio is 3.7 , and the magnetic field compression ratio is also 3.7. The Alfvén Mach number for the shock is $M_{\mathrm{A}}=5.9$. The shock is an almost perfect perpendicular shock with $\theta_{\mathrm{Bn}}=92^{\circ} \pm 2$.

Figure 7 (top panel) shows the magnetic field magnitude at ACE from 04:30-04:40 UT plotted at $12 \mathrm{~s}$ resolution, which shows the arrival of the shock around 04:32 UT. The proton intensity measured by the EPAM instrument (Gold et al. 1998) showed a gradual increase starting late on 26 April, with the $\sim 1 \mathrm{MeV}$ protons rising some four orders of magnitude in intensity up to the arrival of the shock. The EPAM instrument is the spare flight unit for the HI-SCALE detector on Ulysses 

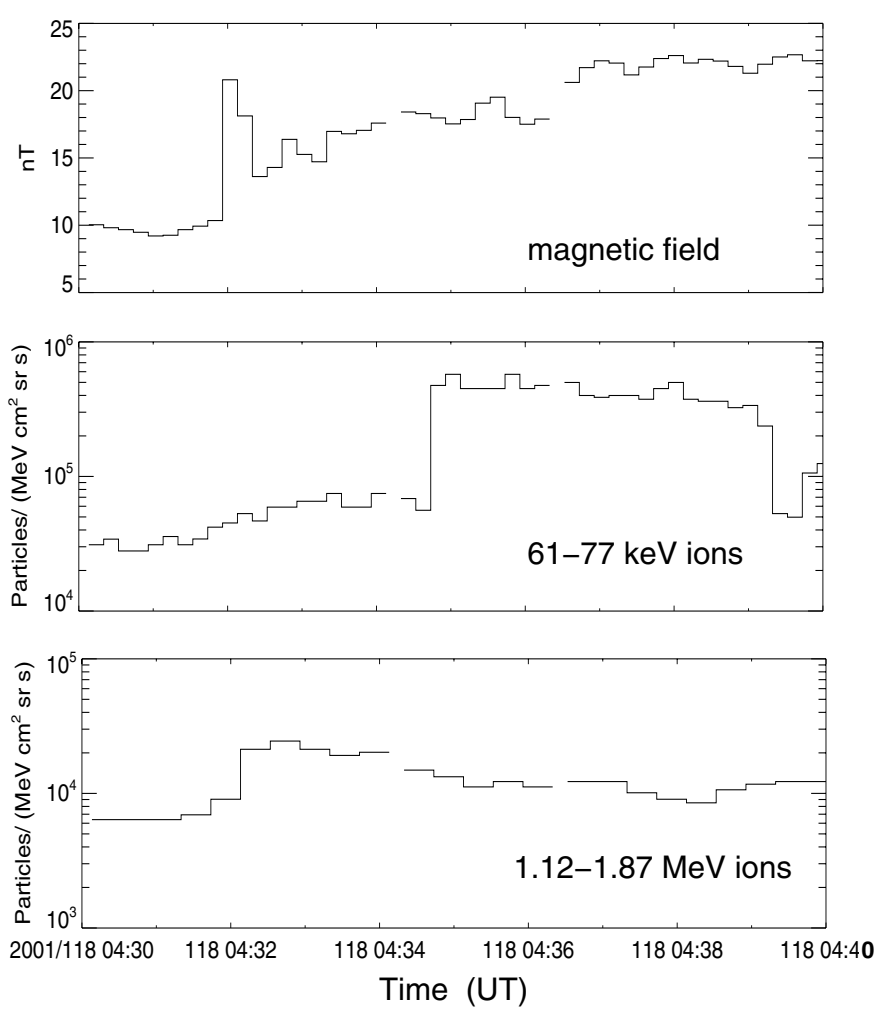

Fig. 7. Top panel: the magnitude of the magnetic field at ACE from 04:30-04:40 UT on 28 April, 2001. The shock arrival is at 04:32 UT. Middle panel: the intensity-time history of $61-77 \mathrm{keV}$ protons from sector 5 of the LEMS120 detector. Lower panel: the intensity-time history of $1.12-1.87 \mathrm{MeV}$ protons from sector 5 of the LEMS120 detector.

and has essentially identical characteristics. Figure 7 (bottom panel) shows the $1.12-1.87 \mathrm{MeV}$ proton intensity in sector 5 of the LEMS120 detector for the same time period, and the same sector in the $61-77 \mathrm{keV}$ protons is shown in the middle panel. The interesting feature of Fig. 7 is the fact that the intensity spike (which is anisotropic) in the $1.12-1.87 \mathrm{MeV}$ ions is after the shock crossing, and therefore the ions are trapped behind the shock. The "rectangular" spike in the 61-77 keV ions (which is fairly isotropic) probably represents the acceleration occurring in the turbulence well behind the shock. The fact that the intensity is not equally high on both sides of the shock suggests that an acceleration mechanism which involves repeated shock crossings is not appropriate. This point therefore supports the type of acceleration mechanism that we are suggesting in this paper.

Figure 8 shows the proton spectrum in sectors 4 and 5 for the period 04:32:20-04:33:32 UT, which covers 6 spacecraft spins. The accumulation times are such that the higher energy channels are at a resolution of two spins. However, the spectral features are seen at the highest time resolution and persist for over a minute. In these two sectors the proton spectrum has a double peak, with the higher one in the $\mathrm{P}^{\prime} 5$ energy channel (336-601 keV) and the lower peak in the $\mathrm{P}^{\prime} 3$ energy channel (127-207 keV).

The EPAM instrument has a high spectral resolution channel (MFSA) which is accumulated with a time resolution

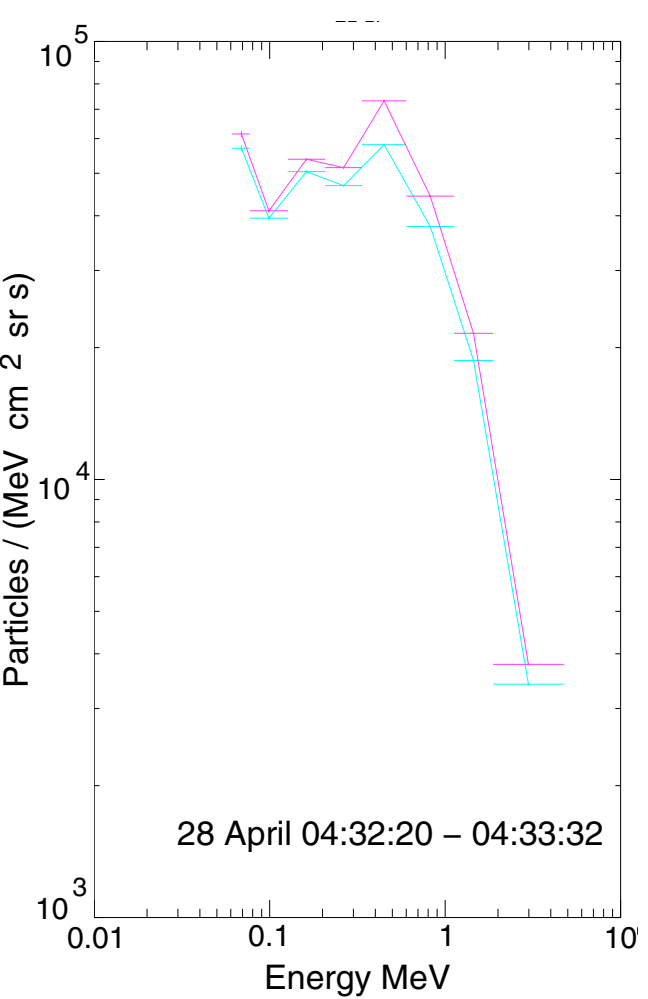

Fig. 8. The proton energy spectrum at the shock, accumulated over the period 04:32:20-04:33:32 UT. The spectral features are seen in sector 5 (higher intensity) and sector 4 of the LEMS120 detector, and the pitch angle distribution (not shown) indicates that these protons are aligned with the magnetic field.

of $1024 \mathrm{~s}$ (see Gold et al. 1998, for details). Thus for periods when the spectrum is not changing rapidly, the MFSA spectrum is a valuable indicator of the true spectrum. Figure 9 shows the spin averaged MFSA spectrum for the period 01:00-02:00 UT on 28 April. There is a broad spectral maximum around $170 \mathrm{keV}$. This feature was clearly visible in the MFSA spectrum accumulated some $14 \mathrm{~h}$ earlier; however, the earlier spectrum was anisotropic, with a forward/backward ratio of over a factor of 10 at around $225 \mathrm{keV}$. As the shock approached ACE, the spectral feature moved slightly lower in energy, and the distribution at the spacecraft became more isotropic. This spectral feature is consistent with the feature seen in the $\mathrm{P}^{\prime} 3$ energy channel in Fig. 8.

As in the 10 May event at Ulysses, there is considerable trapping behind the shock. The proton intensity around $0.1 \mathrm{MeV}$ is around 2 orders of magnitude higher than that seen escaping upstream of the shock four hours before it arrived at ACE. However, at $\sim 2 \mathrm{MeV}$ for the 28 April event there is significant escape, and the intensity-time profile is quite broad around the shock.

\subsection{Electrons}

Both the events we have discussed also had high electron intensities. The intensity-time histories of the 53-103 keV electrons (DE2) for three hours, centred around the shock passage, are shown in Fig. 10, plotted on a linear scale to highlight the 


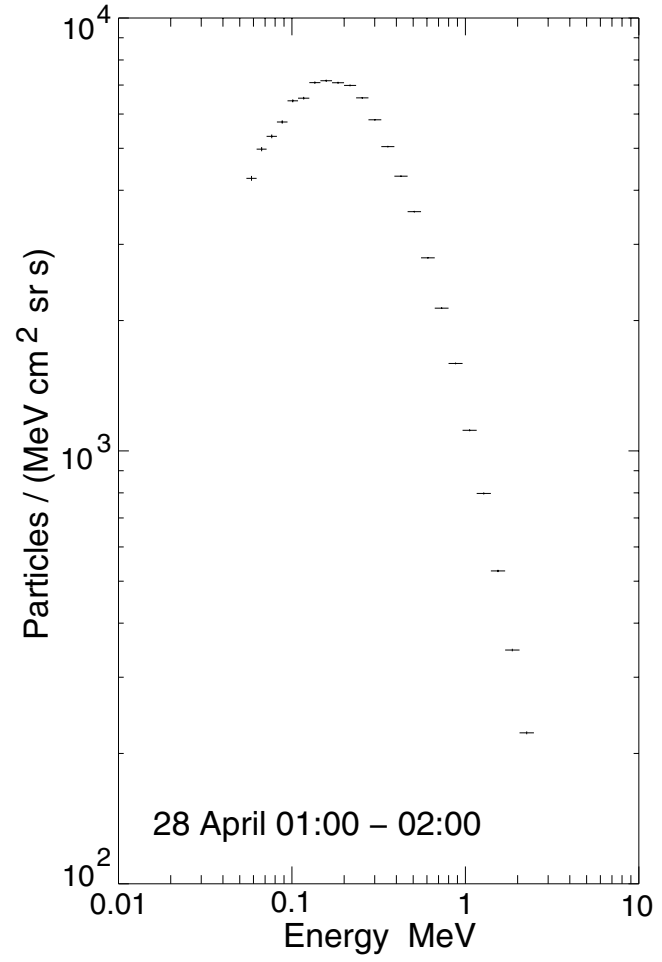

Fig. 9. The proton energy spectrum observed from 01:00-02:00 UT on 28 April, 2001 by the high resolution spectrum accumulator. The spectrum is a sector average.

increase at the shock itself. The dashed lines indicate the arrival time of the shock at the spacecraft; the upstream region is to the left of the line and the downstream region to the right.

The 10 May event at Ulysses did not have a significant (in comparison to the peak intensity) electron intensity emitted upstream of the shock, but the intensity remained fairly high following the shock passage for almost $1 \mathrm{~h}$. The time profile for the 28 April event was different, with electron emission of increasing intensity over $\sim 30 \mathrm{~min}$ before the shock arrival. During this time the pitch angle distribution at ACE is isotropic to better than a factor of 2 . However, following the shock passage, the pitch angle distribution at the period of highest intensity in Fig. 10 is strongly anti-sunward, namely away from the Sun and towards the shock. The pitch angle distribution for the period of highest intensity is shown in Fig. 11.

The solar wind data covering the shock arrival at ACE are shown in Fig. 12. The density increase is very similar to that experienced at Ulysses for the 10 May shock. The speed and temperature increases are within the $64 \mathrm{~s}$ resolution of the data and are also similar to the 10 May shock.

\section{Proton acceleration}

We are now in a position to investigate the likely acceleration process for the protons observed in these events. The 10 May 2001 event is considered first. We evaluate the following physical quantities by use of the observed values before the shock front. These values are: the density $n=2 \mathrm{~cm}^{-3}$, proton temperature $T=3 \times 10^{4} \mathrm{~K}$ (which gives the thermal energy as $2.6 \mathrm{eV}$ ), and the magnetic field $B=6 \mathrm{nT}$. By using these values, we find the Alfvén speed as $V_{\mathrm{A}}=93 \mathrm{~km} \mathrm{~s}^{-1}$, the ion thermal velocity as $C_{\mathrm{s}}=15.8 \mathrm{~km} \mathrm{~s}^{-1}$, the electron cyclotron frequency $\omega_{\text {ce }}=1.05 \times 10^{3} \mathrm{rad} / \mathrm{s}$, and the electron plasma frequency $\omega_{\text {pe }}=7.9 \times 10^{4} \mathrm{rad} / \mathrm{s}$. Then we obtain the plasma beta $\beta=0.028$, and $\omega_{\text {pe }} / \omega_{\text {ce }}=75.2$.

The Alfvén Mach number can be evaluated as $M_{\mathrm{A}}=$ (680-370) $\mathrm{km} \mathrm{s}^{-1} / V_{\mathrm{A}}$ by using the observed flow velocities before and behind the shock, and this gives us $M_{\mathrm{A}}=310 / 93=$ 3.3. This value is probably uncertain by as much $+0.5,-1$, partly because of the change in the shock normal with respect to the magnetic field as the disturbance passes the spacecraft and partly because of uncertainties in the plasma parameters, which affect the Alfvén speed. It is very difficult from a single spacecraft measurement to determine the parameters of a three dimensional structure.

The maximum proton speed obtained by the $\boldsymbol{V}_{\mathrm{p}} \times \boldsymbol{B}$ shock acceleration mechanism is given by the formula as $v=V_{\mathrm{A}}$ $\left(m_{\mathrm{i}} / m_{\mathrm{e}}\right)^{1 / 2}\left(M_{\mathrm{A}}-1\right)^{3 / 2}$. By use of the formula, we find the maximum proton energy to be about $1 \mathrm{MeV}$, although this is very sensitive to the shock Mach number. Nevertheless, for Mach numbers in the 2.3-3.8 range, the maximum energy will be predicted in the few hundred $\mathrm{keV}-$ few $\mathrm{MeV}$ region, which is where it is observed.

We can perform a similar analysis for the 28 April 2001 event. The observed values of the relevant physical parameters before the shock front are: the density $n=3 \mathrm{~cm}^{-3}$, the proton temperature $T=6 \times 10^{4} \mathrm{~K}$ (which gives the thermal energy as $5.2 \mathrm{eV}$ ), and the magnetic field $B=10 \mathrm{nT}$. By using these values, we find the Alfvén velocity as $V_{\mathrm{A}}=126 \mathrm{~km} \mathrm{~s}^{-1}$, the ion thermal velocity as $C_{\mathrm{s}}=22.3 \mathrm{~km} \mathrm{~s}^{-1}$, the electron cyclotron frequency $\omega_{\mathrm{ce}}=1.76 \times 10^{3} \mathrm{rad} / \mathrm{s}$, and the electron plasma frequency $\omega_{\text {pe }}=9.8 \times 10^{4} \mathrm{rad} / \mathrm{s}$.

We regard it as important that both events have a slightly higher spectral feature visible in a specific sector only. This suggests that these shocks are surrounded by significant turbulence, and that there is only a relatively small exit path for the particles accelerated at the shock.

\section{Electron acceleration}

Hoshino \& Shimada (2002) have applied the surfatron mechanism to electrons and they find that the electron acceleration occurs in the shock transition region, which for our purpose may be thought of (in time) as within a minute or so of the shock passage. As in most shock acceleration mechanisms, the critical feature is providing a way of stopping the accelerated particles escaping. Hoshino and Shimada proposed that large amplitude electrostatic solitary waves were responsible, but they concluded that high electron energies were most likely produced when the shock Mach number exceeded several tens. This is not the case for the shocks we have studied here. Figure 10 shows that for both events, the maximum intensity is seen for a few minutes following shock passage. This is well outside the shock itself and thus it suggests that there must be significant trapping over a distance of around $2 \times 10^{5} \mathrm{~km}$ behind the shock. In the faster shock and more perpendicular shock of 28 April, 2001, there was significant escape upstream of the electrons. 

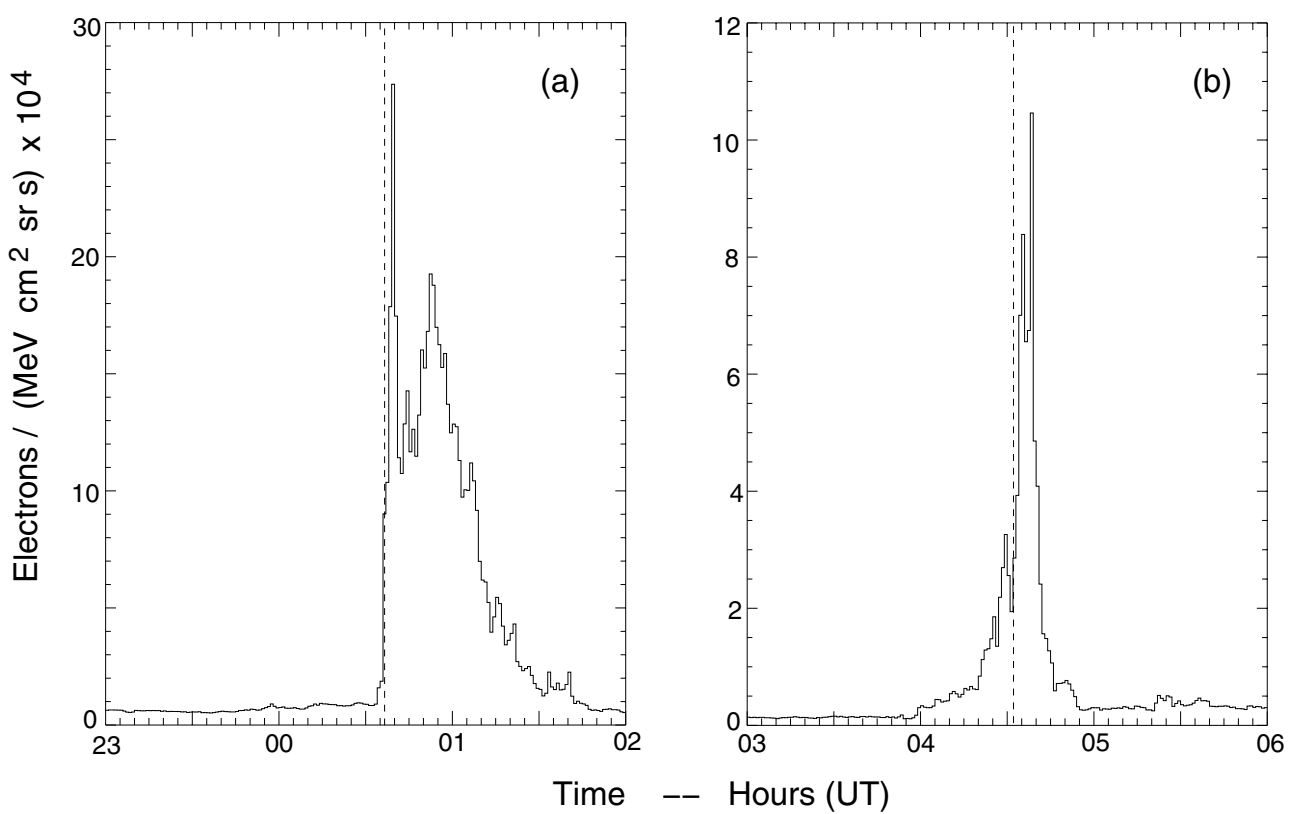

Fig. 10. The 53-103 keV electron intensity-time history for three hours centered around the shock arrival for a) the 10 May 2001 event at Ulysses and b) the 28 April, 2001 event at ACE. The data are plotted as 1 min averages. The times of arrival at the shocks at the spacecraft are indicated by the dashed lines.

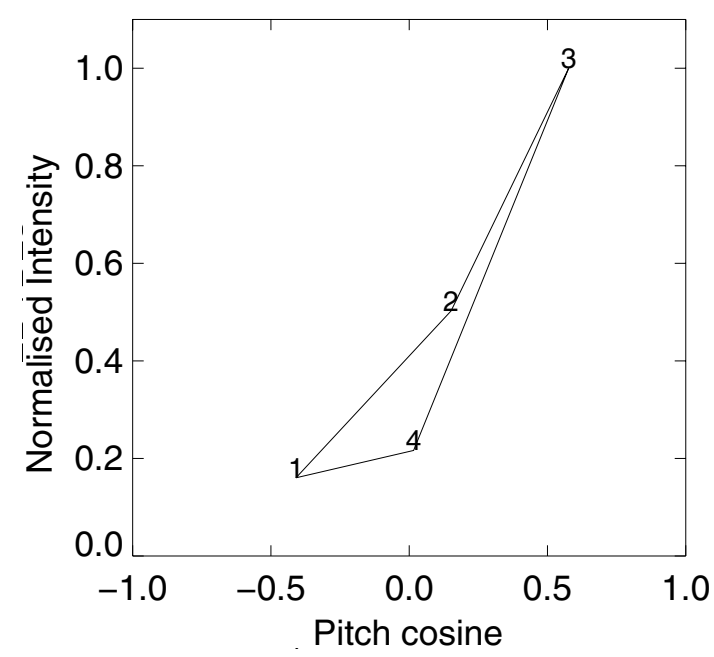

Fig. 11. The 53-103 keV electron pitch angle distribution for the $24 \mathrm{~s}$ interval of peak intensity, 04:38:07-31 UT, following the shock on 28 April, 2001. The numbers on the distribution refer to the sector of the DE2 channel plotted.

\section{Summary}

It is clear that the shock is both accelerating protons and trapping them. If this were not the case the intensity-time profiles would be considerable broader than those observed. There is nevertheless significant escape upstream, especially for the higher energies. (This is not the case for the electrons.)

The compatibility between the theory and observations suggests that the surfatron mechanism outlined by Ohsawa (1985) and Ohsawa \& Sakai $(1987,1988)$ may be a suitable mechanism to describe shock acceleration in the interplanetary medium.
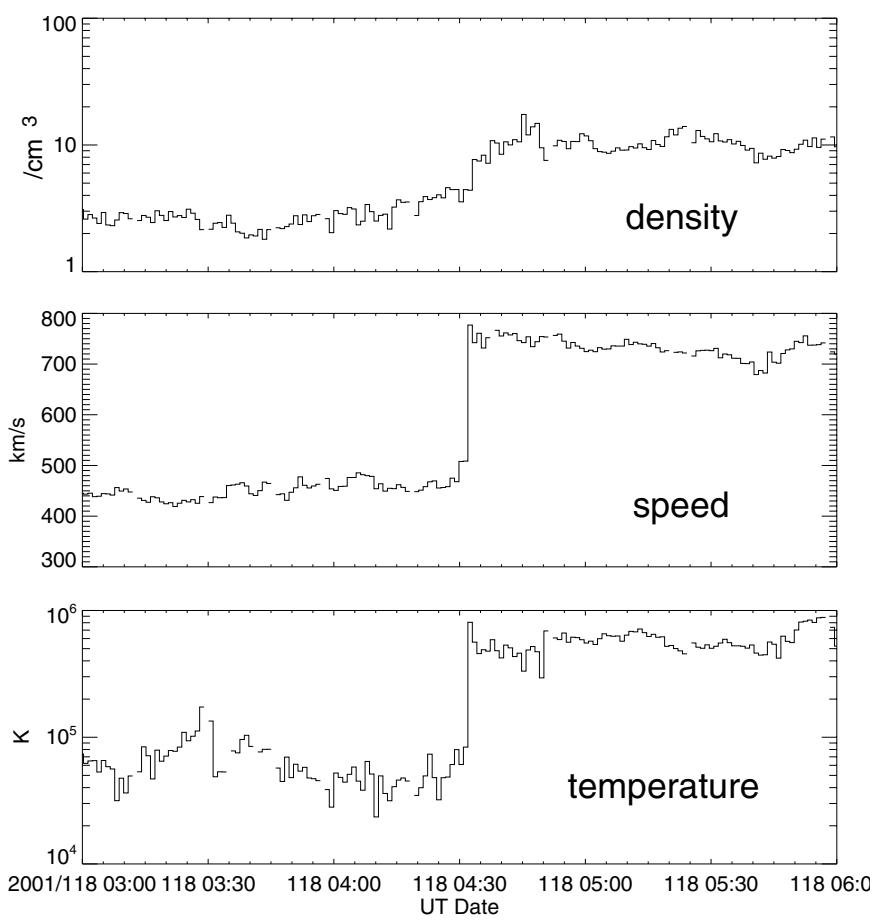

Fig. 12. The solar wind density, speed and temperature at ACE around the time of the shock on 28 April, 2001. These values are taken from the proton population and are $64 \mathrm{~s}$ averages. (Courtesy of Dr. D.J. McComas.)

Acknowledgements. This work was done when one of the authors (Sakai) visited University of Birmingham under the exchange program between the JSPS and the Royal Society. Dr. C. W. Smith provided invaluable guidance on the interpretation of the shocks seen at ACE. Discussions with Drs. S. J. Tappin and I. M. Robinson are greatly 
appreciated. The authors wish to thank their colleagues on the ACE, Ulysses and LASCO teams for their help in making the data available and in a useable form. LASCO is a collaboration between the Naval Research Laboratory, Washington, DC; the Max-Planck-Institute for Aeronomy, Lindau; the Laboratoire d'Astronomie Spatiale, Marseille; and the University of Birmingham. Starlink computing facilities were used for the detailed analysis.

\section{References}

Brueckner, G. E., Howard, R. A., Koomen, M. J., et al. 1995, Sol. Phys., 162, 357

Bougeret, J.-L., Kaiser, M. L., Kellogg, P. J., et al. 1995, Space Sci. Rev., 71, 231
Gold, R. E., Krimigis, S. M., Hawkins, S. E., III, et al. 1998, Space Sci. Rev., 86, 541

Hoshino, M. 2001, Prog. Theor. Phys. Suppl., 143, 149

Hoshino, M., \& Shimada, N. 2002, ApJ, 572, 880

Lanzerotti, L. J., Gold, R. E., Anderson, K. A. et al. 1992, A\&AS, 92, 349

Ogilvie, K. W., \& Arens, J. F. 1971, J. Geophys. Res., 76, 13

Ohsawa, Y. 1985, Phys. Fluids, 28, 2130

Ohsawa, Y., \& Sakai, J.-I. 1985, Geophys. Res. Lett., 12, 617

Ohsawa, Y., \& Sakai, J.-I. 1987, ApJ, 313, 440

Ohsawa, Y., \& Sakai, J.-I. 1988, ApJ, 332, 439

Sagdeev, R. Z., \& Shapiro, V. D. 1973, JETP Lett., 17, 279

Simnett, G. M. 2003, Sol. Phys., 213, 387

Sugihara, R., \& Midzuno, Y. 1979, J. Phys. Soc. Jpn., 47, 1290 